\title{
LX. Crystallographic notes
}

\section{W.J. Lewis M.A.}

To cite this article: W.J. Lewis M.A. (1877) LX. Crystallographic notes, Philosophical Magazine Series 5, 3:20, 453-456, DOI: 10.1080/14786447708639267

To link to this article: http://dx.doi.org/10.1080/14786447708639267

$$
\text { 曲 Published online: } 13 \text { May } 2009 .
$$

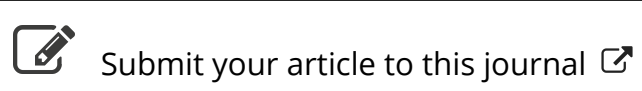

\footnotetext{
Џll Article views: 2
}

Q View related articles $\sqsubset$ 
investigation as to how the special motion assigned to the line of spheres can properly represent the character of the motion of the molecules of a gas in its normal state is my own, no special investigation of this kind being contained in $\mathrm{Mr}$. Waterston's paper. To Mr. Waterston, however, is mainly due the initiative in this subject. As I should be sorry to claim any originality that I did not possess, I would respectfully direct the attention of readers to the portion of $\mathrm{Mr}$. Waterston's paper bearing on this subject.

PS. (2). Professor Clerk Maxwell, to whom this paper was communicated, and who has taken a kindly interest in the subject, has worked out mathematically the velocity for a wave or impulse propagated by a system of particles moving among each other according to the conditions of equilibrium investigated in the first part of this paper-the diameter of the particles being assumed so small as to be negligible compared with their mean distance, and the particles being further assumed spherical, so that there is no movement of rotation developed at the encounters (which would involve loss of velocity).

Under these premises, the velocity of the wave was found to be $\frac{\sqrt{ } 5}{3}$ (or 0.745 ) into the mean velocity of the particles.

In most 'gases the velocity of sound is slightly less than this. This is referable to the movements of rotation developed at the encounters of the molecules (which calculably would delay the wave to a certain extent). In vapour of mercury, according to the determinations of Kundt and Warburg, the velocity of sound is exactly $\frac{\sqrt{ } 5}{3}$ into the molecular velocity.

London, May 1877.

LX. Crystallographic Notes. By W. J. LewIs, M.A., Fellow of Oriel College, Oxford, and Assistant in the Mineral Department, British Museum*.

[Flate IV.]

Barium Nitrate.

TAST autumn my friend Mr. T. Davies, of the British 1 Museum, kindly brought me a fairly large crystal with a very large number of faces on it. It had been found at the

* Communicated by the Crystallological Society. Read April 12,1877. 
bottom of a reagent-bottle which had been put aside for many years. The solution, owing to a faulty stopper, had all evaporated, leaving this single crystal. The label had been lost; so, after a crystallographic investigation, I scraped off a very small portion from a part on which were no crystal-faces. By means of this I was able to determine that the crystal was one of barium nitrate. I have thought that a description of its crystallography would be interesting, both on account of the remarkable development of its faces and its decided tetartohedrism. The facts already known about barium nitrate are that it crystallizes in the cubic system, shows a hemihedrism with parallel faces, and has the forms $\left\{\begin{array}{lll}1 & 0 & 0\end{array}\right\},\left\{\begin{array}{lll}1 & 1 & 1\end{array}\right\}$, and $\pi\{210\}$.

The forms observed on the crystal in question are $a=\{100\}$, $t=\kappa \pi\{124\}, h=\kappa \pi\{124\}, n=\kappa \pi\{531\}, l=\kappa\{311\}$, $s=\kappa\{\overline{2} 11\}, o=\kappa\{\overline{1} 11\}$. A glance at the stereographic projection (fig. 1, Plate IV.) of these forms shows that $t, n$, and $l$ occupy alternate octants, and that $o, s$, and $h$ occupy the remaining octants. The forms $t$ and $h$ are complementary, and make up the hemihedral form with parallel faces $\pi\{124\}$. The physical character of the faces of this form in adjacent octants manifests, however, the tetartohedrism of the crystal; for the faces $h$ are large and smooth, the faces $t$ small and rough. The faces $n$ are tetartohedral and well developed in alternate octants ; they are for the most part bright; but the most careful examination in the remaining octants failed to discover the slightest trace of corresponding planes. Similarly the faces $o$, which are large and bright, were found only in alternate octants. The faces $l$ are fairly developed and bright. The faces $s$ are very small but fairly bright. The faces of the cube $a$ are large and bright.

The principal zones on the crystal are those containing the

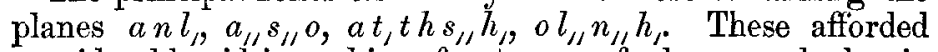
considerable aid in seeking for traces of planes, and also in some instances in determining the real positions of some of the badly developed faces. The following are the most important angles of the combination, with which the measurements accorded well:-

$$
\begin{array}{llllll}
a t, & 29 & 12 & a n & 32 & 19 \\
a t & 77 & 24 & a n, & 80 & 16 \\
a t, & 64 & 7 \frac{1}{2} & a n_{J \prime} & 59 & 32
\end{array} \quad\left[\begin{array}{llll}
o l_{\prime \prime} & 588 & 3 \dot{1} \\
o n_{\prime \prime} & 72 & 58 \frac{1}{2} \\
o h, & 112 & 12 \frac{1}{3}
\end{array}\right.
$$

The stereographic projection (fig. 1) shows very clearly the zones and the arrangement of the poles. Fig. 2 is an orthogonal projection on one of the faces of the cube; and fig. 3 is 
Barium Nitrate.

Fis. 1 .

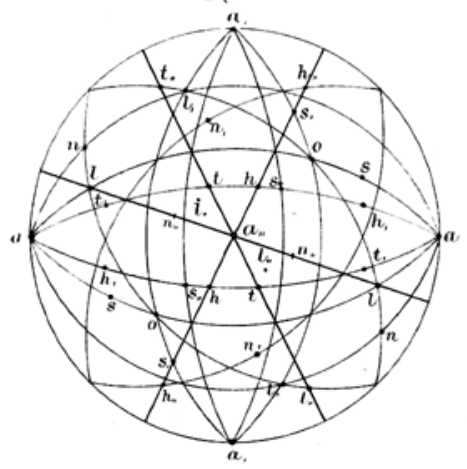

Fig. 2 .

Fig. 3 .

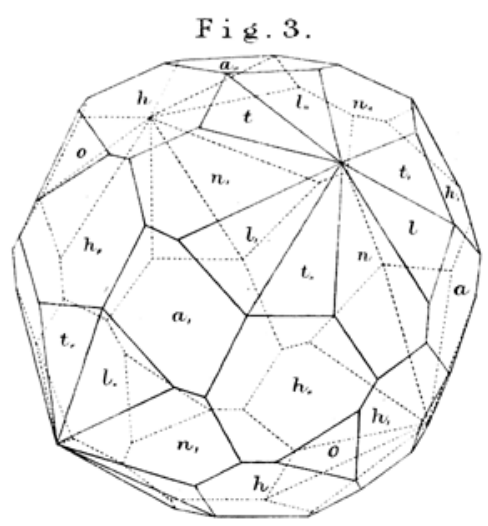

sphene

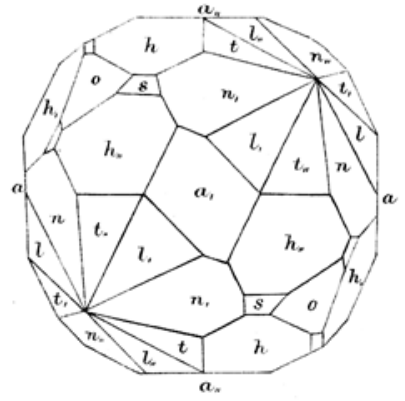

Fig. 34. From Hessenbergs Min. Not.VI.

F i g. 4 .
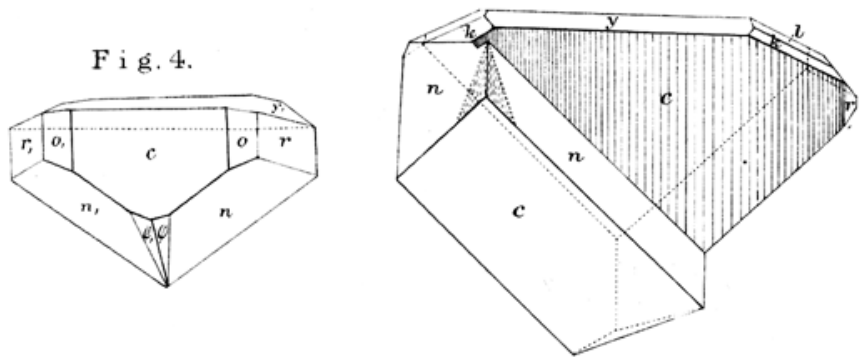

Mintern Bros. Hith. 
one in which the axes have been projected in the usual manner. To avoid confusion, the small planes $s$ have been omitted in the latter figure. The crystal is elongated in a direction nearly coinciding with the normal to a face of the tetrahedron. An oval ring seems to have been first deposited; and on this the crystal has grown, vaulting itself on the lower surface so as not to cling to the bottle. There were no definite crystalfaces to be seen on this concave portion: The top is also irregular and indefinite. The crystal introduced into the polarizing microscope between crossed Nicols depolarized the light. Exact experiments on the rotation of the plane of polarization could not be made without destroying the crystal.

On some crystals of barium nitrate crystallized out of solution during the course of a few weeks, the forms $\kappa\left\{\begin{array}{lll}1 & 1 & 1\end{array}\right\}, \kappa\{\overline{1} 11\}$,

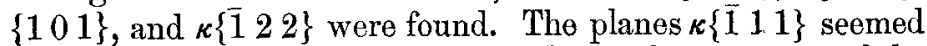
to be smoother and brighter than those of $\kappa\{111\}$; and the edges of the former carried the planes $\kappa\{\overline{1} 22\}$.

\section{Sphene.}

On a crystal from the Tyrol, obtained by me some years ago, two rough ill-developed planes are situated on the quoin formed by the two planes $n=\left\{\begin{array}{lll}1 & 2 & 3\end{array}\right\}$ and the base $c=\left\{\begin{array}{lll}0 & 0 & 1\end{array}\right\}$. They look almost like the result which would be produced by slightly grinding down this quoin. The exact symmetry of the two planes, as also the frequency of their occurrence, show them, however, to be really planes. . Hessenberg, who devoted considerable attention to this mineral, has noticed similar faces on the crystals from the Zillerthal, described in his Min. Notizen, vi., and has introduced them in two of the figures of these crystals. As this part of the Notizen is out of print, I have copied one of these figures (fig. 34), in which the small triangular dotted planes are those under consideration. Hessenberg says that he found them more or less clearly developed on almost all the crystals from this locality: He expresses, however, his conviction that the rough portion is only a continuation of the plane $n$.

Lately I obtained several crystals on which these planes were very fairly developed, of one of which fig. 4 is a projection. They give such excessively bad reflections, that it was only by observing with a ray of sunlight thrown into the room by a mirror, and by slightly oiling the surfaces, that reliable measurements were obtained. The form calculated from these measurements is $\{3,3,10\}$, adopting the axial system given in Miller's 'Mineralogy.' The following are the angles observed and calculated:- 
Observed.

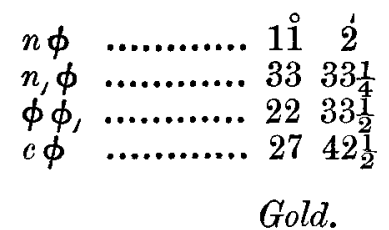

Calculated.

Measurement of a large though imperfect crystal in the British Museum showed it to be a combination of the cube with the tetrakishexahedron $\{410\}$ and the triakisoctahedron $\{811\}$. As the faces were very dull, and but rough measurements could be obtained, I was glad to confirm this observation by the examination of a crystal showing the same combination in Mr. Ludlam's beautiful collection, which he was good enough to lend me. The angles measured on the latter crystal agree fairly well with the calculated angles.

Measured. Calculated.

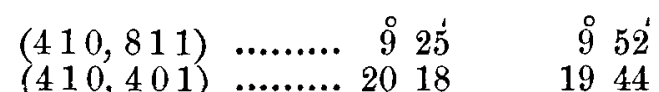

I saw recently a very beautiful crystal of the same combination in the collection of the École des Mines, Paris. In the two former the planes $\{811\}$ are deeply striated parallel to their intersection with the faces of the cube.

LXI. Acoustical Observations.

By LoRd Rayleigh, M.A., F.R.S.*

Perception of the Direction of a Source of Sound.

$\mathrm{T}$ a paper with the above title, communicated last year to 1 the Musical Association and afterwards published in abstract in 'Nature,' I brought forward the fact that we are unable to distinguish whether a pure tone (obtained from a tuning-fork and air-resonator) is immediately in front of or immediately behind us-although with other sounds, and notably with the human voice, there is in general no difficulty. In order to make the experiment satisfactorily, it is necessary to provide two similar forks and resonators and to place the observer between them. At a given signal both forks are struck, but one of them only is held over its resonator. If this precaution be neglected, the noise attending the excitation of the fork vitiates the experiment. Subsequently to the reading of my paper, it occurred to me that if the ordinary

* Communicated by the Author. 